\title{
Reação de cultivares de algodoeiro a Meloidogyne incognita em condições de campo e casa de vegetação no estado de Mato Grosso
}

\author{
Rafael Galbieri ${ }^{1}$, Milton G. Fuzatto ${ }^{2}$, Edivaldo Cia $^{2}$, Reginaldo R. Lüders ${ }^{2}$, Andressa C.Z. Machado ${ }^{3} \&$ \\ Alberto F. Boldt ${ }^{1}$ \\ ${ }^{1}$ Instituto Mato-Grossense do Algodão - IMAmt, 78850-000, Primavera do Leste, MT, Brasil; ${ }^{2}$ Instituto Agronômico de \\ Campinas - IAC, 13020-902, Campinas, SP, Brasil; ${ }^{3}$ Monsanto do Brasil Ltda., 75650-000, Morrinhos, GO, Brasil
}

Autor para correspondência: Rafael Galbieri, e-mail: rafaelgalbieri@imamt.com.br

\begin{abstract}
RESUMO
Genótipos de algodoeiro, compreendendo cultivares e linhagens avançadas, foram avaliados no Estado de Mato Grosso quanto à reação a Meloidogyne incognita. Em dois experimentos de campo, foi avaliada a tolerância a esse nematóide mediante atribuição de notas, de acordo com sintomas externos exibidos pelas plantas. Um ensaio avaliou também a resistência ao parasita, mediante determinação do número de espécimes nas raízes de plantas amostradas nas parcelas. A resistência foi avaliada também em casa de vegetação, por meio da determinação do número de massas de ovos nas raízes e do fator de reprodução. Foram constatadas diferenças notáveis na reação dos 22 genótipos estudados, verificando-se a formação de grupos graduais de desempenho, tanto com respeito à resistência quanto à tolerância. Nas duas características, destacaram-se positivamente as cultivares IAC 25 RMD, IPR 140 e IPR JATAÍ, e negativamente, dentre outras, FIBERMAX 966, LDCV FREGO e FMT 703. A produção de algodão em caroço variou de 482 a 3087 g/parcela no experimento de Campo Verde, e de 1153 a 3151 g/parcela no de Primavera do Leste. As perdas na produção, nos genótipos mais afetados, foram de até 65\% no primeiro experimento e de até $40 \%$ no segundo. Os dados de avaliação da resistência e da tolerância mostraram-se correlacionados, porém, os da tolerância, a julgar pelos coeficientes de variação e de correlação intra-classe, na análise da variância, mostraram-se mais precisos e de maior repetibilidade.
\end{abstract}

Palavras-chave: Gossypium hirsutum, nematóide-das-galhas, genótipos, resistência, tolerância.

\begin{abstract}
Reaction of cotton genotypes to Meloidogyne incognita in field and greenhouse trials in Mato Grosso state, Brazil

Cotton genotypes, comprising cultivars and advanced lineages, were evaluated for tolerance and resistance to Meloidogyne incognita in Mato Grosso state, Brazil. Tolerance was evaluated in two field experiments, attributing scores according to external symptoms exhibited by plants, while resistance was studied in greenhouse and in one field trial, counting the number of egg masses or specimens on the roots, and the reproduction factor. Significant differences were observed among the 22 studied genotypes, concerning both resistance and tolerance, and graduated performance groups were established. The cultivars IAC 25 RMD, IPR 140 and IPR JATAI formed the most resistant/tolerant group, while FIBERMAX 966, LDCV FREGO, and FMT 703, among others, were situated in the most susceptible/intolerant ones. Cotton seed yield ranged from 482 to $3087 \mathrm{~g} / \mathrm{plot}$ at Campo Verde and from 1153 to $3151 \mathrm{~g} / \mathrm{plot}$ at Primavera do Leste. Yield losses, attributable to incidence of this nematode, were estimated at $65 \%$ and $40 \%$, respectively, in these trials. Data related to resistance and to tolerance were correlated, however, and by taking into account coefficient of variation and intra-class correlation, from the analysis of variance, it could be seen that data relating to tolerance presented greater precision and repeatability.
\end{abstract}

Keywords: Gossypium hirsutum, root-knot nematodes, genotypes, resistance, tolerance.

\section{INTRODUÇÃO}

A cada safra, no Estado de Mato Grosso, surgem novas áreas de ocorrência de nematóides no algodoeiro, assim como aumenta o nível de infestação em glebas onde sua presença já fora constatada. Em conseqüência, já se observam consideráveis reduções de produtividade nas lavouras estabelecidas com cultivares suscetíveis. $\mathrm{Na}$ medida que a utilização de tais cultivares é um dos principais fatores para o agravamento do problema (Robinson, 1999), torna-se indispensável a avaliação, quanto à resposta em face desses parasitas, do material disponível para plantio.

Areferida avaliação tem sido feita tanto em condições de campo, sob infestação natural e baseada nos sintomas exibidos pelas plantas, quanto em casa de vegetação, mediante inoculação controlada e quantificação de massa de ovos e cálculo do fator de reprodução. O primeiro método, segundo Cook \& Evans (1987), diz respeito à tolerância ao ataque dos nematóides e teria como vantagem avaliar o desempenho econômico dos genótipos, do ponto de vista 
agrícola, e tendo em conta os demais fatores que ocorrem e interagem nessas condições. A desvantagem, segundo Inomoto (2006), é que genótipos aprovados em função apenas da tolerância, podem permitir a multiplicação e aumento da população do parasita. Outra restrição ao método é que pode haver escapes, devido à possível desuniformidade de distribuição dos nematóides na gleba (Heald et al., 1981). O segundo método avalia, de fato, a resistência, entendida esta como a capacidade do genótipo de impedir ou dificultar a reprodução dos nematóides. Embora tenha sido observada correlação positiva entre esses métodos (Zhou \& Starr, 2003), a possível ocorrência de desencontro entre eles faz com que alguns autores recomendem sua associação, em programas de melhoramento para resistência a esses parasitas (Carneiro et al., 2005).

De acordo com a terminologia exposta, resistência, notadamente em altos níveis, tem sido encontrada, principalmente, em germoplasmas selvagens e em linhagens parentais utilizadas em programas de melhoramento (Robinson, 1999). Seriam poucas, todavia, as cultivares efetivamente utilizadas nas lavouras, que dispõe desse atributo (Koenning et al, 2001; McPherson et al., 2004). Por outro lado, o que se pode considerar ser pelo menos tolerância, está presente, em níveis adequados, tanto em linhagens como em cultivares que, de longa data, vêm sendo postas à disposição dos cotonicultores (Robinson, 1999; Cia et al., 2007; Fuzatto \& Cia, 2001; Cavaleri \& Gridi-Papp, 1993). O presente trabalho teve por objetivo avaliar a resposta de cultivares e linhagens avançadas de algodoeiro à incidência de Meloidogyne incognita (Mi), quanto à tolerância/resistência, em condições de campo e em casa de vegetação, no Estado de Mato Grosso.

\section{MATERIAL E MÉTODOS}

\section{Experimentos em campo}

Foram realizados dois experimentos, um em Primavera do Leste e outro em Campo Verde, Mato Grosso. No primeiro, o solo apresentava $29 \%$ de argila e $60 \%$ de areia, e a gleba vinha sendo cultivada há quatro anos, os três últimos com o algodoeiro. No segundo (solo com $40 \%$ de argila e $49 \%$ de areia), a área se encontrava no terceiro ano de cultivo, os dois últimos com o algodoeiro.

Com delineamento em blocos ao acaso, com sete repetições em Primavera do Leste e 14 em Campo Verde, foram estudados os seguintes genótipos: BRS 01-56818, BRS ARAÇÁ, BRS BURITI, BRS CEDRO, COODETEC 02-1367, COODETEC 406, COODETEC 408, COODETEC 409, DELTAOPAL, FIBERMAX 966, FIBERMAX 993, FMT 701, FMT 703, IAC 25 RMD, IPR 02-307, IPR 140, IPR JATAÍ, LDCV FREGO, LDCV 2, LDCV 5, LDCV 9 e NUOPAL. As parcelas experimentais foram constituídas por uma linha, com seis metros de comprimento, no espaçamento de $0,90 \mathrm{~m}$. No experimento de Primavera do Leste foi intercalada entre as parcelas uma linha com a cultivar FIBERMAX 966, sabidamente sensível ao ataque de nematóides (Cia et al., 2007). A semeadura foi realizada em 04/12/2007, e 30 dias após a emergência foi efetuado desbaste, deixando-se oito plantas por metro. No expeirmento de Primavera do Leste foram empregados no plantio $200 \mathrm{~kg} / \mathrm{ha}$ da fórmula 3-30-0 e, em cobertura, $560 \mathrm{~kg} / \mathrm{ha}$ de fórmula 20-0-20. Das mesmas fórmulas, foram utilizados, em Campo Verde, $350 \mathrm{~kg} / \mathrm{ha}$ no plantio (08/12/2007) e $400 \mathrm{~kg} / \mathrm{ha}$ em cobertura.

A avaliação para nematóides foi realizada 60 dias após a semeadura, atribuindo-se às parcelas notas de 1 a 5 , levando em conta o desenvolvimento das plantas e a presença de clorose típica nas folhas ("carijó"), conforme o seguinte critério: nota 1 - plantas com desenvolvimento normal e sem sintomas foliares; nota 2 - desenvolvimento normal e poucas plantas (até 10\%) com folha "carijó" no baixeiro, ou plantas sem sintomas foliares, porém, com o porte levemente reduzido em algumas plantas; nota 3 - desenvolvimento normal mas com a maioria das plantas exibindo sintomas nas folhas do baixeiro, ou plantas sem sintomas, porém, com o desenvolvimento medianamente afetado na maioria delas; nota 4 - plantas com desenvolvimento reduzido, a maioria delas com sintomas, em algumas atingindo as folhas do ponteiro; nota 5 - todas as plantas com porte fortemente reduzido, com sintomas sobretudo no ponteiro.

Em Campo Verde, aos 60 dias após a emergência, foram coletados, em sete repetições, os sistemas radiculares de seis plantas, localizadas em pontos eqüidistantes situados nos três metros centrais da parcela. As raízes foram processadas pelo método proposto por Coolen \& D' Herde (1972), para quantificação de ovos $+\mathrm{J}_{2}$ de Mi.

Por fim, foi determinada nas parcelas a produção de algodão em caroço e os dados obtidos, assim como os referentes às notas de avaliação e de contagens, transformados, respectivamente, em $\sqrt{x+1}$ e $\log (x+1)$, foram submetidos à análise da variância, efetuando-se teste de agrupamento de médias pelo método de Scott \& Knott. Utilizando-se o método proposto por Fuzatto et al. (2007), foram estimadas as perdas possíveis na produção, decorrentes do uso dos genótipos mais suscetíveis.

\section{Experimento em casa de vegetação}

Os mesmos 22 genótipos foram estudados quanto à resistência ao nematóide, em casa de vegetação provida de sistema automático de resfriamento Pad Argila, com temperatura regulada para $27 \pm 2^{\circ} \mathrm{C}$. O delineamento foi de blocos ao acaso, com 15 repetições, cada parcela constituída por uma planta mantida em copo plástico de $500 \mathrm{~mL}$, contendo $400 \mathrm{~mL}$ de substrato composto por $50 \%$ de areia, $25 \%$ de argila, acrescido de $1 \%$ de matéria orgânica, $\mathrm{pH}=5,9$ e desinfestado com brometo de metila (150 mL de $\mathrm{CH}_{3} \mathrm{Br}$ por $1.000 \mathrm{~L}$ de substrato). Como inóculo utilizou-se mistura de duas populações de Mi raça 3, uma proveniente de Vargem Grande do Sul SP e outra de Campo Verde MT, multiplicadas em tomate (Lycopersicon esculentum "Rutgers") por 60 dias. A semeadura ocorreu em 15/02/2008 e a inoculação em 11/03/2008, em dois 
orifícios com $5 \mathrm{~cm}$ de profundidade, distantes $2,5 \mathrm{~cm}$ do colo da planta, nos quais foram depositados 3.000 ovos + juvenis de segundo estádio $\left(\mathrm{J}_{2}\right)$ de Mi por planta. Decorridos 50 dias da inoculação, as raízes das plantas foram lavadas, pesadas e imersas em solução de floxina B $0,015 \%$ por 10 minutos (Taylor \& Sasser, 1978), quantificando-se, em seguida, o número de massas de ovos de Mi por grama de sistema radicular. $\mathrm{O}$ fator de reprodução (FR) foi calculado mediante soma do número de Mi nas raízes, processadas de acordo com Coolen \& D'Herd (1972), e no solo (Jenkins, 1964), e divisão pela concentração inicial do inóculo (Pi = 3000). Tanto os dados de números de massas de ovos, transformados em $\sqrt{x+1}$, como de FR, transformados em $\log (\mathrm{x}+1)$, foram submetidos à análise de variância e ao teste de agrupamento de médias, de acordo com Scott \& Knott, a $5 \%$ de significância.

\section{RESULTADOS E DISCUSSÃO}

Os resultados obtidos no experimento de Campo Verde revelam diferenças notáveis entre os genótipos, qualquer que seja a variável analisada (Tabela 1). As notas médias atribuídas aos sintomas variaram de 1,15 a 4,47, do que resultou a formação de seis grupos distintos de tolerância. Variação ainda mais pronunciada foi observada com respeito à produção de algodão em caroço, com a formação de sete grupos de genótipos e diferença de 540\% na produção, entre os extremos. Embora com formação de apenas três grupos de genótipos, é acentuada a diferença entre eles também com respeito à quantidade de espécimes do nematóide presente nas raízes de plantas amostradas nas parcelas. As correlações (Tabela 4) entre produção e notas para sintomas $\left(\mathrm{r}=-0,93^{* *}\right)$ e entre produção e número de

TABELA 1 - Reação de genótipos de algodoeiro a Meloidogyne incognita sob condições de campo em Campo Verde MT, no ano agrícola 2007/08

\begin{tabular}{|c|c|c|c|c|c|c|c|c|}
\hline \multirow{2}{*}{$\begin{array}{l}\text { Genótipos } \\
\text { IAC } 25 \text { RMD }\end{array}$} & \multicolumn{2}{|c|}{$\operatorname{Notas}^{(1)}$} & \multirow{2}{*}{$\begin{array}{c}\text { Genótipos } \\
\text { IAC } 25 \text { RMD }\end{array}$} & \multicolumn{2}{|c|}{ Espécimes (2) } & \multirow[t]{2}{*}{ Genótipos } & \multicolumn{2}{|c|}{ Produção ${ }^{(3)}$} \\
\hline & 1,15 & $a^{(4)}$ & & 50,4 & $\mathrm{a}^{(4)}$ & & 3087 & $\mathrm{a}^{(4)}$ \\
\hline IPR 140 & 1,26 & $\mathrm{a}$ & IPR JATAÍ & 135,5 & $\mathrm{~b}$ & IAC 25 RMD & 2596 & $\mathrm{~b}$ \\
\hline IPR JATAÍ & 1,53 & $\mathrm{~b}$ & IPR 140 & 138,9 & $\mathrm{~b}$ & FMT 701 & 2504 & $\mathrm{~b}$ \\
\hline FMT 701 & 1,63 & $\mathrm{~b}$ & BRS CEDRO & 150,7 & $\mathrm{~b}$ & BRS CEDRO & 2308 & $\mathrm{c}$ \\
\hline BRS CEDRO & 1,77 & $\mathrm{~b}$ & COODETEC 409 & 168,8 & $\mathrm{~b}$ & IPR JATAÍ & 2222 & $\mathrm{c}$ \\
\hline COODETEC 409 & 2,05 & $\mathrm{c}$ & LDCV 2 & 190,7 & $\mathrm{~b}$ & COODETEC $02-1637$ & 2158 & $\mathrm{c}$ \\
\hline IPR 02-307 & 2,12 & $\mathrm{c}$ & COODETEC 408 & 196,1 & $\mathrm{~b}$ & COODETEC 409 & 2103 & $\mathrm{c}$ \\
\hline COODETEC $02-1637$ & 2,12 & $\mathrm{c}$ & NUOPAL & 196,7 & $\mathrm{~b}$ & BRS BURITI & 1788 & $\mathrm{~d}$ \\
\hline DELTAOPAL & 2,25 & $\mathrm{c}$ & FMT 701 & 196,7 & $\mathrm{~b}$ & NUOPAL & 1783 & $\mathrm{~d}$ \\
\hline FIBERMAX 993 & 2,29 & $\mathrm{c}$ & COODETEC 02-1637 & 212,0 & $\mathrm{c}$ & BRS ARAÇÁ & 1760 & $d$ \\
\hline NUOPAL & 2,39 & $\mathrm{c}$ & BRS ARAÇÁ & 214,7 & $\mathrm{c}$ & IPR 02-307 & 1680 & d \\
\hline BRS ARAÇÁ & 2,43 & $\mathrm{c}$ & FIBERMAX 993 & 241,7 & $\mathrm{c}$ & LDCV 9 & 1590 & e \\
\hline BRS 01-56818 & 2,47 & $\mathrm{c}$ & IPR 02-307 & 248,0 & $\mathrm{c}$ & LDCV 2 & 1589 & $\mathrm{e}$ \\
\hline LDCV 9 & 2,62 & $\mathrm{~d}$ & BRS 01-56818 & 273,6 & $\mathrm{c}$ & FIBERMAX 993 & 1545 & $\mathrm{e}$ \\
\hline COODETEC 408 & 2,67 & $\mathrm{~d}$ & LDCV 5 & 278,5 & $\mathrm{c}$ & BRS 01-56818 & 1530 & e \\
\hline LDCV 2 & 2,70 & $\mathrm{~d}$ & LDCV 9 & 280,9 & $\mathrm{c}$ & DELTAOPAL & 1450 & e \\
\hline COODETEC 406 & 2,77 & $\mathrm{~d}$ & DELTAOPAL & 284,1 & $\mathrm{c}$ & COODETEC 408 & 1332 & $\mathrm{e}$ \\
\hline BRS BURITI & 2,88 & $\mathrm{~d}$ & LDC FREGO & 323,4 & $\mathrm{c}$ & COODETEC 406 & 1296 & $\mathrm{e}$ \\
\hline LDCV 5 & 3,41 & $\mathrm{e}$ & COODETEC 406 & 334,3 & $\mathrm{c}$ & FMT 703 & 1106 & $\mathrm{f}$ \\
\hline FMT 703 & 3,68 & $\mathrm{e}$ & BRS BURITI & 369,8 & $\mathrm{c}$ & LDCV 5 & 856 & $\mathrm{f}$ \\
\hline FIBERMAX 966 & 4,44 & $\mathrm{f}$ & FMT 703 & 380,0 & $\mathrm{c}$ & FIBERMAX 966 & 660 & $\mathrm{~g}$ \\
\hline LDC FREGO & 4,47 & $\mathrm{f}$ & FIBERMAX 966 & 460,0 & $\mathrm{c}$ & LDC FREGO & 482 & $\mathrm{~g}$ \\
\hline Media & 2,50 & & & 242,1 & & & 1701 & \\
\hline "F" TRAT & 47,23 & $* *$ & & 3,29 & $* *$ & & 37,07 & $* *$ \\
\hline C.V. \% & 7,0 & & & 15,0 & & & 7,0 & \\
\hline$r_{I}{ }^{(5)}$ & 0,89 & & & 0,25 & & & 0,84 & \\
\hline
\end{tabular}

(1) Notas de 1 a 5, crescentes com a intensidade dos sintomas; nota 1 - plantas com desenvolvimento normal e sem sintomas foliares; nota 2 - desenvolvimento normal e poucas plantas (até 10\%) com folha "carijó" no baixeiro, ou plantas sem sintomas foliares, porém, com o porte levemente reduzido em algumas plantas; nota 3 - desenvolvimento normal mas com a maioria das plantas exibindo sintomas nas folhas do baixeiro, ou plantas sem sintomas, porém, com o desenvolvimento medianamente afetado na maioria delas; nota 4 - plantas com desenvolvimento reduzido, a maioria delas com sintomas, em algumas atingindo as folhas do ponteiro; nota 5 - todas as plantas com porte fortemente reduzido, com sintomas sobretudo no ponteiro.

${ }^{(2)}$ Número de espécimes por grama de raiz;

${ }^{(3)}$ Produção de algodão em caroço, em g/parcela;

(4) Teste de Scott \& Knott, a 5\% de significância;

(5) Correlação Intra Classe 
espécimes nas raízes $\left(\mathrm{r}=-0,78^{* *}\right)$, não deixam dúvidas quanto ser a ação predominante desse nematóide a causa do desempenho produtivo altamente diferenciado dos genótipos. A estimativa da perda possível na produção, atribuível à incidência desse parasita, foi de $65 \%$, nos genótipos mais afetados. Assinale-se que uma análise realizada 60 dias após a semeadura revelou 500 espécimes de $\mathrm{Mi} / 200$ cc de solo, na área experimental.

A Tabela 2 mostra os resultados obtidos no experimento de Primavera do Leste. De início, deve-se ressaltar a alta e uniforme infestação com nematóides na gleba, constatada pelo exame das marginais intercalares às parcelas, constituídas pela cultivar FIBERMAX 966, que receberam exclusivamente notas 4 e 5 na avaliação. Como no experimento anterior, foi notável a diferença entre os genótipos com respeito às notas médias para sintomas, as quais variaram de 1,11 a 4,79 , resultando na constituição de cinco grupos de tolerância. Da mesma forma, as produções de algodão em caroço diferiram em até $173 \%$, com os genótipos classificados em quatro grupos. A correlação entre produção e sintomas $(\mathrm{r}=-0,84 *$ *) também aqui confirma o efeito prejudicial desse parasita, no desempenho produtivo dos genótipos. A propósito, nos genótipos mais afetados, a perda possível na produção, atribuível ao ataque do nematóide, foi estimada em $40 \%$. Ressalte-se que análise de amostra da gleba revelou 400 espécimes de Mi/200 cc de solo, aos 50 dias após a semeadura.

Uma análise de variância conjunta dos dados de notas para sintomas nos dois experimentos, revelou um valor $\mathrm{F}=25,4^{* *}$, C.V. $=10,9 \%$ e correlação intra-classe $\mathrm{r}_{\mathrm{I}}=0,92$. Pelo Teste de Scott \& Knott, os genótipos foram classificados em quatro grupos de tolerância. Ademais, a correlação entre as notas atribuídas nos dois experimentos foi de $r=0,93^{* *}$. Esses resultados, bem como os dos experimentos individuais, mostram alta consistência e repetibilidade dos dados, e, associados às correlações antes mencionadas, envolvendo a produção de algodão, comprovam a confiabilidade da avaliação da tolerância,

TABELA 2 - Reação de cultivares de algodoeiro a Meloidogyne incognita sob condições de campo em Primavera do Leste MT, no ano agrícola 2007/08

\begin{tabular}{|c|c|c|c|}
\hline Genótipos & Notas ${ }^{(1)}$ & Genótipos & Produção ${ }^{(2)}$ \\
\hline IAC 25 RMD & $1,11 \mathrm{a}^{(3)}$ & IPR 140 & $3151 \mathrm{a}^{(3)}$ \\
\hline IPR JATAÍ & 1,14 a & IAC 25 RMD & 3106 a \\
\hline IPR 140 & 1,14 a & BRS CEDRO & 3019 a \\
\hline FMT 701 & 1,26 a & COODETEC 02-1637 & 3015 a \\
\hline COODETEC 409 & 1,29 a & FMT 701 & 2994 a \\
\hline COODETEC $02-1637$ & 1,51 a & IPR JATAÍ & $2680 \mathrm{~b}$ \\
\hline BRS 01-56818 & 1,66 a & COODETEC 409 & $2574 \mathrm{~b}$ \\
\hline BRS ARAÇÁ & $1,81 \quad b$ & LDCV 2 & $2399 \mathrm{c}$ \\
\hline DELTAOPAL & $2,00 \mathrm{~b}$ & IPR 02-307 & $2399 \mathrm{c}$ \\
\hline NUOPAL & $2,06 \mathrm{~b}$ & NUOPAL & $2244 \mathrm{c}$ \\
\hline COODETEC 408 & $2,07 \quad b$ & FIBERMAX 993 & $2237 \mathrm{c}$ \\
\hline LDCV 2 & $2,09 \mathrm{~b}$ & BRS BURITI & $2222 \mathrm{c}$ \\
\hline IPR 02-307 & $2,17 \quad b$ & COODETEC 408 & $2207 \mathrm{c}$ \\
\hline BRS CEDRO & $2,23 \mathrm{~b}$ & COODETEC 406 & $2109 \mathrm{c}$ \\
\hline FIBERMAX 993 & $2,41 \quad b$ & LDCV 9 & $2101 \mathrm{c}$ \\
\hline LDCV 9 & $2,47 \quad b$ & DELTAOPAL & $2053 \mathrm{c}$ \\
\hline COODETEC 406 & $2,74 \mathrm{c}$ & BRS ARAÇÁ & $2004 \mathrm{c}$ \\
\hline BRS BURITI & $2,83 \mathrm{c}$ & BRS 01-56818 & $1974 \mathrm{c}$ \\
\hline FMT 703 & $3,57 \mathrm{~d}$ & FMT 703 & $1959 \mathrm{c}$ \\
\hline LDCV 5 & $3,81 \mathrm{~d}$ & LDCV 5 & $1471 \mathrm{~d}$ \\
\hline LDC FREGO & $4,04 \mathrm{~d}$ & LDC FREGO & $1357 \mathrm{~d}$ \\
\hline FIBERMAX 966 & $4,79 \mathrm{e}$ & FIBERMAX 966 & $1153 \mathrm{~d}$ \\
\hline Media & 2,28 & & 2292 \\
\hline "F" TRAT & $15,83 \quad * *$ & & $15,16 * *$ \\
\hline C.V. $\%$ & 10,0 & & 16,0 \\
\hline$r_{I}^{(4)}$ & 0,51 & & 0,50 \\
\hline
\end{tabular}

\footnotetext{
(1) Notas de 1 a 5, crescentes com a intensidade dos sintomas; nota 1 - plantas com desenvolvimento normal e sem sintomas foliares; nota 2 - desenvolvimento normal e poucas plantas (até 10\%) com folha "carijó" no baixeiro, ou plantas sem sintomas foliares, porém, com o porte levemente reduzido em algumas plantas; nota 3 - desenvolvimento normal mas com a maioria das plantas exibindo sintomas nas folhas do baixeiro, ou plantas sem sintomas, porém, com o desenvolvimento medianamente afetado na maioria delas; nota 4 - plantas com desenvolvimento reduzido, a maioria delas com sintomas, em algumas atingindo.

(2) Produção de algodão em caroço, em g/parcela;

(3) Teste de Scott \& Knott, a 5\% de significância;

(4) Correlação Intra Classe.
} 
para prever o desempenho agrícola de genótipos, na presença desse nematóide. Vale notar que os resultados desses experimentos - tanto com respeito à amplitude de variação das notas, como com relação ao desempenho de alguns dos genótipos neles estudados - estão de acordo com os obtidos por Cia et al. (2007).

$\mathrm{Na}$ Tabela 3 encontram-se os dados obtidos no experimento em casa de vegetação. Tal como nos experimentos feitos em campo, foi substancial a diferença entre os genótipos, nas duas características avaliadas. No caso do número de massas de ovos por raiz, foram constituídos cinco grupos de resistência e quanto ao fator de reprodução, três grupos foram diferenciados. Uma correlação com $\mathrm{r}=$ $0,85^{* *}$ entre os dados mostrou equivalência entre os dois métodos para avaliar a resistência de genótipos a esse nematóide. Apesar de discrepâncias, no caso de alguns genótipos, há correlação entre os dados obtidos com esses métodos e aqueles resultantes da avaliação da tolerância em campo, como também apontaram Cook \& Evans (1987). É de se notar, entretanto, que essas correlações foram bem menores do que a verificada entre notas referentes aos sintomas, nos dois experimentos de campo, e mesmo do que a observada entre notas e contagens de espécimes nas raízes, no experimento de Campo Verde (Tabela 4).
Com respeito a desempenhos adequados - levando em conta as notas e produções obtidas, e a análise de grupamento das médias - pode-se considerar como altamente tolerante a IAC 25 RMD e como tolerantes IPR 140, IRP JATAÍ e FMT 701. Com base na massa de ovos/raiz e no fator de reprodução, e excluída a FMT 701, essa classificação pode ser aplicada também com relação à resistência.

É conveniente ressaltar que, nos dois métodos de avaliação, coincidem os genótipos que integraram os grupos mais extremos de resistência/tolerância e de suscetibilidade/ intolerância. Isso, se não elimina, pelo menos atenua as restrições à tolerância como critério de seleção e avaliação de genótipos, com respeito a nematóides, feitas, dentre outros, por Davis \& May (2005). Vale notar, entretanto, que nos grupos de reação intermediária podem ocorrer discrepâncias, como a verificada com a linhagem IPR 02-307, que revelou tolerância razoável, mas resistência sofrível. Ou, situação inversa, como a observada com a COODETEC 406, que apresentou resistência possivelmente aceitável, mas tolerância - e, conseqüentemente, produção - certamente rejeitáveis. Quanto aos métodos, em si, embora tenham sido ambos eficientes, é válido notar que, além de mais trabalhosa e dependente de instalações e procedimentos

TABELA 3 - Reação de cultivares de algodoeiro a Meloidogyne incognita sob condições de casa de vegetação, em Primavera do Leste MT, no ano agrícola de 2008

\begin{tabular}{|c|c|c|c|c|}
\hline Genótipos & $\begin{array}{c}\text { Número de massas de } \\
\text { ovos/raiz }\end{array}$ & Genótipos & $\begin{array}{r}\text { Fator de } \\
\text { Reproduçã }\end{array}$ & $\begin{array}{l}\text { e } \\
\text { ção }\end{array}$ \\
\hline IAC 25 RMD & $0,6 \mathrm{a}^{(1)}$ & IAC 25 RMD & 0,5 a & $a^{(1)}$ \\
\hline IPR 140 & $5,5 \mathrm{~b}$ & IPR JATAÍ & $1,1 \quad \mathrm{~b}$ & $\mathrm{~b}$ \\
\hline IPR JATAÍ & $8,8 \mathrm{c}$ & IPR 140 & $1,2 \mathrm{~b}$ & $\mathrm{~b}$ \\
\hline COODETEC 406 & $9,9 \mathrm{c}$ & COODETEC 406 & $1,2 \mathrm{~b}$ & $\mathrm{~b}$ \\
\hline COODETEC 408 & $11,1 \quad \mathrm{c}$ & COODETEC $02-1637$ & $1,4 \mathrm{~b}$ & $\mathrm{~b}$ \\
\hline COODETEC 409 & $11,1 \quad \mathrm{c}$ & COODETEC 409 & $1,5 \mathrm{~b}$ & $\mathrm{~b}$ \\
\hline BRS CEDRO & $11,8 \quad \mathrm{c}$ & LDCV 2 & $1,6 \mathrm{~b}$ & $\mathrm{~b}$ \\
\hline BRS ARAÇÁ & $14,8 \quad \mathrm{c}$ & FMT 701 & $1,7 \mathrm{~b}$ & $\mathrm{~b}$ \\
\hline FMT 701 & $15,2 \mathrm{c}$ & BRS CEDRO & $1,8 \mathrm{~b}$ & b \\
\hline COODETEC 02-1637 & $17,3 \mathrm{c}$ & COODETEC 408 & $2,0 \quad \mathrm{~b}$ & $\mathrm{~b}$ \\
\hline LDCV 2 & $18,1 \quad \mathrm{c}$ & BRS ARAÇÁ & $2,1 \quad b$ & $\mathrm{~b}$ \\
\hline LDCV 5 & $18,9 \mathrm{~d}$ & BRS BURITI & $2,4 \quad \mathrm{c}$ & $\mathrm{c}$ \\
\hline NUOPAL & $19,2 \mathrm{~d}$ & LDC FREGO & $2,4 \quad c$ & $\mathrm{c}$ \\
\hline IPR 02-307 & $23,8 \mathrm{~d}$ & DELTAOPAL & $2,6 \mathrm{c}$ & $\mathrm{c}$ \\
\hline BRS BURITI & $25,1 \quad d$ & NUOPAL & $2,7 \mathrm{c}$ & $\mathrm{c}$ \\
\hline FMT 703 & 27,0 e & LDCV 5 & $2,7 \mathrm{c}$ & $\mathrm{c}$ \\
\hline BRS 01-56818 & 28,8 e & BRS 01-56818 & $2,8 \mathrm{c}$ & $\mathrm{c}$ \\
\hline LDCV 9 & 29,0 e & FIBERMAX 993 & $3,0 \quad \mathrm{c}$ & $\mathrm{c}$ \\
\hline DELTAOPAL & 29,1 e & FMT 703 & $3,1 \quad \mathrm{c}$ & $\mathrm{c}$ \\
\hline LDC FREGO & 29,8 e & IPR 02-307 & $3,8 \quad \mathrm{c}$ & $\mathrm{c}$ \\
\hline FIBERMAX 993 & 30,3 e & FIBERMAX 966 & $4,0 \quad \mathrm{c}$ & $\mathrm{c}$ \\
\hline FIBERMAX 966 & $34,2 \mathrm{e}$ & LDCV 9 & $4,4 \quad \mathrm{c}$ & $\mathrm{c}$ \\
\hline Media & 19,1 & & 2,3 & \\
\hline "F" TRAT & $9,34 * *$ & & $6,07 *$ & $* *$ \\
\hline C.V. $\%$ & 35,0 & & 46,0 & \\
\hline $\mathrm{r}_{\mathrm{I}}^{(2)}$ & 0,36 & & 0,25 & \\
\hline
\end{tabular}

(1) Teste de Scott \& Knott, a 5 \% de significância;

(2) Correlação Intra Classe. 
TABELA 4 - Correlação entre variáveis estudadas nos experimentos em campo e em casa de vegetação, realizados com Meloidogyne incognita, em Mato Grosso em 2007/08

\begin{tabular}{lccccccc}
\hline \hline & \multicolumn{3}{c}{ Campo $^{(1)}$} & & \multicolumn{2}{c}{ Casa de vegetação } \\
\cline { 2 - 4 } \cline { 7 - 8 } & N CV & PRO PV & PRO CV & & MO & FR \\
\hline N PV & $0,93 * *$ & $-0,84 * *$ & --- & & $0,64 * *$ & $0,61 * *$ \\
E CV & $0,84 * *$ & --- & $-0,78 * *$ & & $0,78 * *$ & $0,68 * *$ \\
PRO CV & $-0,93 * *$ & $0,94 * *$ & --- & & --- & --- \\
N CV & --- & --- & --- & & $0,68 * *$ & $0,57 * *$ \\
MO & --- & --- & --- & & --- & $0,85 * *$ \\
\hline
\end{tabular}

(1) Variáveis: $\mathrm{N} \mathrm{CV}=$ notas em Campo Verde; E CV = espécimes em Campo Verde; PRO CV = produção de algodão em Campo Verde; $\mathrm{N}$ $\mathrm{PV}=$ notas em Primavera do Leste; $\mathrm{PRO}$ PV $=$ produção em Primavera do Leste;

(2) $\mathrm{MO}=$ número de massas de ovos; FR = fator de reprodução.

especiais, a avaliação da resistência - quer se considere o coeficiente de variação, quer se leve em conta a correlação intra-classe, nas análises estatísticas - mostrou-se menos precisa e de menor grau de repetibilidade (Tabelas 1, 2 e 3). Por outro lado, pode-se argumentar que a eficiência da avaliação da tolerância, depende essencialmente de ocorrer alta e uniforme infestação dos nematóides, na gleba utilizada, como aconteceu nos presentes experimentos. $\mathrm{O}$ uso conjunto dos dois métodos, obviamente, deve resultar em avaliações mais eficazes e seguras.

\section{AGRADECIMENTOS}

Ao Fundo de Apoio à Cultura do Algodão - FACUAL e Associação Mato-Grossense dos Produtores de Algodão - AMPA pelo apoio financeiro para a realização desse trabalho.

\section{REFERÊCIAS BIBLIOGRÁFICAS}

Carneiro RMDG, Neves DI, Falcão R, Paes NS, Cia E, Gossi de SÁ MF (2005) Resistência de genótipos de algodoeiro a Meloidogyne incognita raça 3: reprodução e histopatologia. Nematologia Brasileira 299:1-10.

Cavaleri PA, Gridi-Papp IL (1993). Algodão. In: Furlani, AMC, Viegas GP (Eds.) O Melhoramento de Plantas no Instituto Agronômico, v.1. Campinas SP. Instituto Agronômico. pp. 13-27.

Cia E, Galbieri R, Fuzatto MG, Lüders RR, Kondo JI, Carvalho
LH, Ruano O, Almeida WP, Ito MF, Oliveira AB, Cunha HF, Chiavevato EJ, Aguiar PU, Mehta YR, Martins ALM, Pettinelli Junior A, Bolonhezi, D, Foltran DE, Kasai FS, Ito MA, Michelotto MD, Bortoletto N, Gallo PB, Reco PC, Souza PS, Rossetto R, Freitas RS, Furlani Junior E, Lebedenco A, Pedrosa MB, Lanza MA (2007) Comportamento de genótipos de algodoeiro na presença de patógenos e nematóides. Revista Brasileira de Oleaginosas e Fibrosas 11:99-109.

Coolen WA, D'Herde CJ (1972) A method for the quantitative extraction of nematodes from plant tissue. Ghent. State Agricultural Research Centre.

Cook R, Evans K (1987) Resistance and tolerance. In: Brown RH; Kerry (Eds.) Principles and practice of nematode control in crops. Marrickville, NSW, Australia. Academic Press. pp. 179-231.

Davis RF, May OL (2005) Relationship between yield potential and percentage yield suppression caused by the southern root-knot nematode in cotton. Crop Science 45:2312-2317.

Fuzatto MG, Cia E, Lüdes RR, Galbieri R (2007) Metodologia para estimativa de perdas devidas a doenças em experimentos com genótipos de algodoeiro. Anais, 6. Congresso Brasileiro de Algodão. Campina Grande PB. Embrapa CNPA. CD ROM.

Fuzatto MG, Cia E (2001). Algodoeiro: Novas cultivares IAC destacam-se pela resistência a doenças. O Agronômico 53:19-20

Heald CM, Birchfield W, Blackmon CW, Hussey RS, Orr CC, Shepherd RL, Veech J, Smith FH (1981) Nematodes. In: Watkins GM (Eds.) Compendium of cotton disease. Saint Paul MN. APS Press. pp. 50-56.

Inomoto MM (2006) Nematóides e seu controle. In: Moresco E (Ed.) Algodão - Pesquisas e Resultados para o Campo. Vol. 2, Cuiabá MT. Fundo de Apoio à Cultura do Algodão. pp. 241-261.

Jenkins WR (1964) A rapid centrifugal-flotation technique for separation nematodes from soil. Plant Disease Reporter 48:692.

Koenning SR, Barker KR, Bowman, DT (2001) Resistance as a Tactic for Management of Meloidogyne incognita on Cotton in North Carolina. Journal of Nematology 33:126-131.

McPherson MG, Jenkins JN, Watson CE, McCarty, Jr C (2004) Inheritance of Root-knot Nematode Resistance in M-315 RNR and M78- RNR Cotton. The Journal of Cotton Science 8:154161.

Robinson AF (1999) Cotton Nematodes. In: Smith CW, Cothren JT (Eds.) Cotton: Origin, History, Tecnology and Production. New York NY. John Wiley and Sons. pp. 595-615.

Taylor AL, Sasser JN (1978) Biology, identification and control of root-knot nematodes (Meloidogyne sp.). Raleigh NC. North Carolina State University.

Zhou E, Starr JL (2003) A comparison of the damage functions, root galling, and reproduction of Meloidogyne incognita on resistant and susceptible cotton cultivars. The Journal of Cotton Science 7:224-230. 